\title{
A CASE OF ACTINOMYCOSIS OF THE OVARY AND PELVIC CONNECTIVE TISSUE.*
}

\author{
BY D. BERRY HART, M.D., \\ Obstetric Physician to the Royal Maternity and Simpson Memorial Hospital ; Gynæ- \\ cologist to the Royal Infirmary, Edinburgh.
}

THE case I wish to discuss with you is probably one of the rarest possible in Gynæcology, and is thus open to the obvious criticism of being a somewhat unsuitable one for consideration before an audience most of whom are rightly anxious to learn what they must meet, diagnose, and treat in practice, and not what interests the lecturer from its mere rarity. With this feeling I quite sympathize, and am not, as a rule, disposed to consider before you only what is uncommon. I have broken through my rule to-day for the following reasons: In the first place, I did not diagnose the case, and made what one might euphemistically term the next best diagnosis-viz., pelvic tubercular disease-but really formed what is better described as an erroneous opinion of the woman's malady.

A diagnosis not having been arrived at, my treatment was unsatisfactory, and my anxiety was not soothed by the fact that the exact nature of the case was not settled at the post-mortem, but only after the specimens removed had been examined microscopically and the peculiar fungus found. The course of this case, as it slowly dragged on, was briefly as follows:

Mrs. H., forty-nine, married and nulliparous, was admitted to Ward 36 on August 23, Igor, complaining of swelling on the left side (iliac region), and weakness. In June she began to feel weak, and had to remain in bed during July. There was a good deal of downbearing pain in the bowels, and constipation. In August she had sweatings, and began to get thinner. Her sexual history was unimportant. She had married a year ago, and the menopause had occurred in March of this year.

In my absence, Dr. Milne Murray saw her, and felt a swelling at the side of the uterus, which he thought might be tubal. When I returned, the physical signs had altered, as there could now be felt through the left lateral and posterior fornices a firm flat effusion exactly like the exudation in early puerperal cellulitis. Dr. Murray also noticed this alteration, and it was valuable to have his previous observations, as the small swelling could not now be felt. The

* A Clinical Lecture given at the Royal Infirmary, January 7 , I902. 


\section{Journal of Obstetrics and Gynæcology}

patient's general condition was unsatisfactory, as she was a feeble, run-down woman.

In September she developed phlegmasia alba of the left leg; there was a good deal of pain, requiring morphia to ease it; there was also an evening rise of temperature to $101^{\circ}-102^{\circ} \mathrm{F}$. Had this condition followed a labour or abortion, the diagnosis would unhesitatingly have been puerperal cellulitis going on to pus formation. There was no history, however, of this; the woman was forty-nine and past the menopause, and this special diagnosis could not be entertained.

I could not make a diagnosis, but my opinion was that it might be malignant, for the following reasons: The pain and swelling in the leg favoured this idea, but the temperature was against it, as well as the absence of any involvement of the inguinal glands through the anastomosis between them and the pelvic lymphatic glands, viâ the one lying on the obturator membrane. The thickening felt through the fornices decreased, and I thus had to give up this opinion as an untenable one.

In the middle of October the temperature became higher, and rigors occurred. I examined all the regions where pus might point, but neither in the hip, iliac fossa, fornices, or lumbar regions could I detect any such attempt. There was an cedema for some time in the groin, but no pus. There was distinct thickening just below the ilio-pectineal eminence, but the dissection necessary to get at this point, or the hysterectomy one might have done in a younger woman, seemed to me too dangerous in this case.

I finally made up my mind that the case might be tubercular, going on to abscess. Tubercle may occur at this age (I have seen it in a woman of seventy-five), and upon the whole this seemed the most feasible explanation, although I was far from satisfied with it.

On November I, I opened the abdomen, and felt the swelling at and below the left ilio-pectineal eminence, but it did not diffuse far, and was not spreading into the iliac fossa. Had it done so I would have made an extraperitoneal incision near the iliac spine, and pushed my fingers extraperitoneally to the mass in the way I have found useful in other cases. The pelvic organs were matted and indistinguishable.

The patient seemed somewhat better after this, but her strength soon failed, and she passed blood in her urine, and had some purulent discharge per vaginam. The urine was ordered to be centrifuged and examined microscopically, but by some mistake this was not done. This was a most unfortunate circumstance, as you will soon realize. 
The poor woman died on November I8, and, fortunately, a postmortem was obtained. This was as follows :

Thorax.-Pleurisy on both sides.

Heart.-No special change.

Abdomen.-Chronic adhesion of pelvic viscera. On left side of pelvis and up to kidney below peritoneum a large amount of blood and pus.

Bladder contained a pint of recent blood-clot.

The rectum was eroded and perforated; septic thrombosis in pelvic veins.

Ovary (left).-Flabby, soft, and apparently suppurative.

The impression this examination made on me as to clearing up of the case was most unsatisfactory. The idea of its being a mere pelvic abscess I could not entertain, as it lacked etiology, and then the hæmorrhage was a most unusual thing. I had never seen this occur in an ordinary cellulitic suppuration, and so far as I have read I have never met with such a case.

Dr. Welsh, however, when the ovary was examined, found the ray fungus, Actinomycosis bovis; and this, of course, cleared up the case at once, and gave out the belated diagnosis of actinomycosis of the ovary and pelvic connective tissue, with secondary infection of the liver, lungs, etc.

We may now discuss the following points:

I. The nature of actinomycosis so far as it concerns us here.

2. A criticism of the opinion and treatment.

3. Some general questions as to diagnosis.

I. The Nature of Actinomycosis so far as it concerns us here.This disease affects the lower animals-oxen, horses, pigs-more than the human species. Bollinger, in 1877 , discovered it in the lower animals, and Israel, in 1878 , in man.

In the ox it affects mainly the jaw, tongue, and palate. In the human species it enters by the mouth, jaw, osophagus, intestine, lungs, skin, genital tract in the female, and rectum.

The actinomyces form little colonies, often yellowish in colour, visible in the pus to the naked eye. The actinomyces is one of the higher bacteria, and in the colonies we find branches, filaments, cocci, and clubs.

The filaments are protoplasmic, and have a sheath, branch markedly, and interlace.

The cocci are produced from growths arising from the filaments.

The clubs are seen at the margin, and seem to be produced by a 


\section{Journal of Obstetrics and Gynæcology}

hyaline swelling of the sheath of the end of a filament. They may not be seen in the human species. This parasite grows outside the body, on barley usually, and this explains the lesion in the lower animals-viz., it is acquired by mastication or inhalation. In the human species it may arise in the same way, or may be inoculated by using straw in cleansing the parts after defæcation.

When once introduced it spreads slowly, causing suppuration, hæmorrhage, metastases to lung, liver-in fact, pyæmia.

MacFadyean believes that the cocci start the growth, and are carried by leucocytes or in plasma; these elongate and form short bacillary forms and the long filaments. The clubs are due to degenerative changes in the filaments.

2. Criticism of the Opinion and Treatment.-In this case I formed no diagnosis. When we do so, we mean that the case is seen through, understood completely. We cannot speak of a wrong or right diagnosis; diagnosis must be right. It is like a 'bull's-eye,' which needs no qualifying adjective. The term 'opinion' is a good one, and should be used when one does not form a diagnosis. We can thus have a right or wrong opinion; an opinion can be changed, but not a diagnosis. This may seem pedantic, but it is useful, and keeps one cautious. No one should be satisfied if only an opinion has been formed, and careful examinations should go on to obtain certainty.

I was certain in this case that it was not an ordinary cellulitis ending in pelvic abscess. It had some of the features of pelvic abscess-situation, temperature, rigors-but the patient was too seriously ill at first; there was no child-bearing history, the age was against it.

I thought first of malignant disease. It had some of its features -induration, pain, swelling of left leg-but the temperature was not that of malignant disease. There was no affection of the inguinal glands, such as one gets in late malignant disease of the pelvic connective tissue, and rigors are not a feature of malignancy.

There remained two things: tubercular disease and actinomycosis.

Tubercular disease seemed a likely thing. The age was not against it at all, neither was the temperature or rigors and evident pus formation. I adopted this, therefore, as my opinion, but with a reservation that there was more in the case, and that I had not formed a diagnosis.

I did not think of Actinomycosis. I had never seen a case, although I had read of it, and you will find it mentioned and 
described in the 'Manual of Gynæcology' (Hart and Barbour). You see, I had the knowledge, but did not apply it. I might have got light had I said, 'Now, what else might cause this condition?' and if I had looked up even what I had already written.

I certainly would have made a diagnosis had I examined the pus or the urine. This was an indefensible omission. I had no right to assume this was an ordinary fotid urine from catheterism.

You see, gentlemen, I failed to ascertain all the facts, and the result was unsatisfactory to me, and especially to the patient.

I can only say she was very ill all the time, and I hesitated to do an extensive operation in her weak state. I think now I should have risked it had the patient consented.

I am standing in the white sheet so that you may learn from it how unsatisfactory it is in such a case to be without a diagnosis, and how serious our work is. Had I attained to a diagnosis, who knows what drainage and iodide of potash might have done?

3. Some General Points as to Diagnosis. - This case shows you the importance of diagnosis. With it you can treat the case, or if you do not know what that should be, you can look up authorities. Your books are not so available, however, if you have not your diagnosis as an index. Remember that disease sometimes unfolds itself slowly, and it is quite one thing to diagnose fully developed conditions, and a much more difficult matter to make out obscure ones slowly dragging on in their evolution.

It is a good rule to diagnose before you operate, but in abdominal cases you have sometimes to operate in order to diagnose. This case certainly belonged to the latter category. 